\title{
Use of lithium and cancer risk in patients with bipolar disorder: population-based cohort study
}

\author{
Ru-Yu Huang, * Kun-Pin Hsieh, * Wan-Wen Huang and Yi-Hsin Yang
}

\section{Background}

Lithium inhibits glycogen synthase kinase-3, which is an enzyme involved in the pathogenesis of cancer.

\section{Aims \\ To investigate the association between lithium and cancer risk in patients with bipolar disorder.}

\section{Method}

A retrospective cohort study was designed using the National Health Insurance Research Database (NHIRD) in Taiwan. Patients using lithium comprised the index drug group and patients using anticonvulsants only comprised the control group. Time-dependent Cox regression was used to evaluate the hazard ratios (HRS) for risk of cancer

\section{Results}

Compared with anticonvulsant-only exposure, lithium exposure was associated with significantly lower cancer risk $(\mathrm{HR}=0.735,95 \% \mathrm{Cl}$ 0.554-0.974). The hazard ratios for the first, second and third tertiles of the cumulative defined daily dose were $0.762(95 \% \mathrm{Cl} 0.516-1.125), 0.919(95 \% \mathrm{Cl}$ $0.640-1.318)$ and $0.552(95 \% \mathrm{Cl} 0.367-0.831)$, respectively.

\section{Conclusions}

Lithium is associated with reduced overall cancer risk in patients with bipolar disorder. A dose-response relationship for cancer risk reduction was observed.

\section{Declaration of interest}

None.

\section{Copyright and usage}

(c) The Royal College of Psychiatrists 2016.
Glycogen synthase kinase-3 (GSK-3) is a serine/threonine kinase known to be involved in multiple cellular processes, including signalling transduction, gene transcription, translation, cytoskeletal organisation in cell-cycle progression and survival. Because of its multiple functions, GSK-3 plays important roles in many human disorders such as cancer, immune disorders, metabolic disorders, neurodegenerative diseases and neuropsychiatric diseases. ${ }^{1}$ Consequently, GSK-3 inhibitors are speculated to be potential agents for treatment. The role of GSK-3 in cancer development remains complex and controversial. ${ }^{2}$ GSK-3 may play a positive role in cell proliferation and its aberrant expression as a tumour promoter. Several studies have shown that GSK-3 is overexpressed in various tumour types, such as colon, liver, ovarian and pancreatic tumours. ${ }^{3}$ However, GSK-3 may also be a tumour suppressor. GSK-3 can suppress the Wnt/ $\beta$-catenin pathway by phosphorylating $\beta$-catenin, which results in the degradation of $\beta$-catenin. ${ }^{2,3}$ Many target genes of $\mathrm{Wnt} / \beta$-catenin signalling are proto-oncogenes that have been directly implicated in cancer development. Furthermore, increased $\beta$-catenin levels have been reported to be linked to various types of cancers, including colorectal cancer, melanoma, hepatocellular and ovarian cancers, whereas conflicting data exist for prostate cancer. Another pathway related to cancer development is aberrant activation of the Hedgehog signalling pathways. It has been reported that the Hedgehog pathway is linked to basal cell carcinomas, medulloblastoms and rhabdomyosarcomas. ${ }^{1}$ Lithium, primarily used for bipolar disorder and as augmentation therapy for refractory depression through two possible mechanisms of action, was discovered as the first inhibitor of GSK-3 in $1996 .{ }^{4}$ Lithium is an excellent mood stabiliser for the treatment of bipolar disorder (the gold standard), and provides several benefits: prevents both manic and depressive episodes and exerts acute antimanic and antisuicidal effects. ${ }^{5}$ A hospital-based cohort study has reported a slightly, but not statistically significant, higher total

*These authors contributed equally to the work. cancer prevalence rate in participants taking lithium compared with those in the control group. ${ }^{6}$ However, in their analysis based on histopathological category, a lower but non-significant risk of developing non-epithelial tumours was found in the lithium group compared with the control group. We found very few clinical studies that have investigated the association between cancer risk and lithium. The aim of our study was to investigate lithium usage and cancer risk in the population. Additionally, we estimated the dose-response relationship of lithium usage with its cumulative defined daily dose, cumulative prescription days and average daily dose.

\section{Method}

\section{Data sources}

A retrospective cohort study was conducted based on Taiwan's National Health Insurance Research Database (NHIRD). The single-payer National Health Insurance programme was launched in Taiwan in 1995, and it has enrolled more than $99 \%$ of the 23 million people in Taiwan's population. The NHIRD contains claim records of the beneficiaries, such as demographic data, prescriptions and expenditure for healthcare services. All investigators are required to sign an agreement that guarantees patient confidentiality before using the database. The accuracy of diagnoses of major diseases in the NHIRD, such as stroke, has been validated. ${ }^{7}$ We used the Longitudinal Health Insurance Database 2005 (LHID 2005), a subset of the NHIRD, which comprises a randomly sampled representative database of 1000000 people from the entire NHI enrolees who were alive in 2005. There were no statistically significant differences in the distribution of gender and age or average insured payroll-related amount between the sample group of the LHID and the original NHIRD. ${ }^{8}$ This study was approved by the Institutional Review Board of Kaohsiung Medical University Hospital, Kaohsiung, Taiwan (KMUHIRB-EXEMPT(I)-20150034). 
Participants who have been diagnosed with bipolar disorder extracted from the Longitudinal Health Insurance Database 2005 during 1998-2009 (ICD-9-CM: 290.0-296.1, 296.4-296.9) $(n=17972)$

Exclusion:

(a) Age $<18$ or age $\geqslant 100(n=788)$

(b) Never used index drug (lithium or anticonvulsants) $(n=10034)$

(c) Cancer diagnosis prior to index drug use $(n=393)$

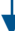

6757 patients divided into three groups: anticonvulsants only; lithium only; and ever exposure to lithium/anticonvulsants

Exclusion:

(a) Anticonvulsants prior to the index date in the anticonvulsantonly group $(n=1730)$

(b) Follow-up time $<1$ year $(n=298)$

Follow-up to the earliest date of any cancer diagnosis, death, disenrolment or the end of $2010(n=4729)$

Fig. 1 Diagram for identifying study participants.

\section{Study cohort}

Patients were identified by a diagnosis of bipolar disorder (ICD-9-CM codes 296.0, 296.1, and 296.4-296.9) between 1998 and 2009.9 Lithium was the primary index drug. To identify a comparison group with the same indications for lithium, users of anticonvulsants were selected as controls because of their comparable antimanic and antidepressant properties, and reimbursement period in the Taiwan NHI programme. ${ }^{10,11}$ We further excluded patients younger than 18 years old, the extremely elderly (older than 100 years), those with less than 1 year of follow-up and those with a diagnosis of cancer before the index date. The earliest date of lithium or anticonvulsant exposure was defined as the index date. Study cohorts were divided into three groups: anticonvulsants only, lithium only and ever exposure to anticonvulsants and lithium. The end of the observation period was the date of cancer diagnosis, death, disenrolment from the NHI programme or the end of 2010, whichever occurred first.

\section{Drug exposure and outcome measurement}

Anatomical therapeutic chemical classification system (ATC) codes were used to identify medications (lithium: N05AN01; anticonvulsants: N03AF01, N03AF02, N03AG01, N03AX09, N03AX11, N03AX12). ${ }^{12}$ The defined daily dose (DDD) recommended by the World Health Organization (WHO) is the assumed average maintenance dose per day for a drug used for its main indication in adults. Cumulative DDD (cDDD) was calculated by summing the total DDD during the study period. Cumulative prescription days were measured by accumulating the days of lithium prescriptions for all visits within the study period. Average daily dose was expressed as cDDD divided by cumulative prescription days. Dose-response relationships regarding incidence were evaluated in three categories based on the amount of CDDD and average daily DDD: low (less than $33 \mathrm{rd}$ percentile), medium (between 33rd and 66th percentile) and high (above the 66th percentile).

As the ICD-9-CM diagnosis may be coded for disease-screening purposes in the NHI medical claims database, cancer diagnosis was confirmed by the Registry for Catastrophic Illness (ICD-9-CM codes for malignant neoplasm: 140-208). To be included in the Registry, patient medical records have to be reviewed and approved according to pathology evaluation and/or cytology evidence for exemption from copayments for the treatment of the disease. It includes approximately $90-92 \%$ of cancer patients in the Taiwan Cancer Registry.

\section{Covariates}

Analysis covariates included gender, age at the index date, comorbidities and comedications. Baseline comorbidities were defined as any appearance of corresponding ICD-9-CM codes in the in-patient or out-patient records during the 12 months prior to the index date. Diseases included those listed in the Deyo-Charlson Comorbidity Index, which summarises 17 diagnostic categories ${ }^{13}$ (online Table DS1). Some diseases with a higher prevalence in patients with bipolar disorder than in the general population were also considered, including other acute or chronic ischaemic diseases, hypertension, arrhythmias, dyslipidaemia and obesity. In addition, psychiatric comorbidities comprised schizophrenia, other psychosis, depression, anxiety disorders, alcohol-related disorders and substance-related disorders ${ }^{14}$ (online Table DS1).

Baseline comedications were identified by prescriptions dispensed during the 6 months prior to the index date, including metformin, sulfonylureas, aspirin, non-steroidal anti-inflammatory drugs (NSAIDs), cyclo-oxygenase-2 (COX-2) inhibitors, statins, systemic steroids, angiotensin-converting enzyme inhibitors (ACEIs), angiotensin II receptor blockers (ARBs), antidepressants (tricyclic antidepressants (TCAs), selective serotonin reuptake inhibitors (SSRIs) and other antidepressants), first-generation antipsychotics (FGAs), second-generation antipsychotics (SGAs) and anxiolytics/hypnotics ${ }^{15}$ (online Table DS2).

\section{Statistical analysis}

For between-group comparisons, $t$-tests or ANOVAs were used for continuous variables, and chi-squared tests or Fisher's exact tests were used for nominal variables.

Logistic regression was used to estimate the predicated probability (propensity score) of the treatment drug for each patient, including the covariates of baseline comorbidities and comedication (online Tables DS1 and DS2). ${ }^{16}$ Inverse probability of treatment weights (IPTW) were calculated as the inverse of the estimated propensity score for the lithium group and the inverse of one minus the estimated propensity score for the control group. ${ }^{17}$ The IPTW was applied to the regression analysis to create a pseudo-population with a similar covariate distribution of individual treatment groups. The Cox regressions were used to estimate hazard ratios (HRs) and their 95\% confidence intervals for the occurrence of cancer and for the various dose-response categories. Two models were implemented to investigate effects from various groups of covariates. Model I was weighted by IPTW. Model II additionally included cancer-risk-related drugs. All the analysis variables in the Cox regressions were examined for possible violation of proportional hazards assumptions, and time-dependent covariates were added if the assumption was not met.

The crude incidence for different types of cancer was calculated for individuals on lithium with and without anticonvulsant use and for anticonvulsant-only users. The numerator for the incidence was number of patients with each type of cancer during follow-up, and the denominator was the number of person-years of follow-up. All data management and statistical analyses were 
performed using SAS 9.4 software. All statistical tests in this study were two-sided. $P<0.05$ was considered statistically significant. A post hoc power analysis was also conducted using the POWER procedure in SAS.

\section{Sensitivity and subgroup analyses}

We also conducted several sensitivity analyses. First, we broadened the definition for cancer diagnosis to at least one ICD-9-CM code in the claims database. Although cancer development may need a longer time than 10 years, and although sometimes ICD-9 coding was given for screening purposes, this definition could be a better representation of suspected early cancer signs. Second, we only included prescriptions of lithium or anticonvulsants prescribed by psychiatrists. Because anticonvulsants may have other indications, such as epilepsy and neuropathic pain, this restriction reduces confounding. Third, patients with an epilepsy diagnosis prior to the index date were excluded to investigate the potential confounding of other indications for anticonvulsants. ${ }^{18}$

Combined therapies are common in bipolar disorder treatments. They are often used to discontinue or shift to another medication if the patient cannot tolerate the side-effects of the drug. Although the association between valproate and cancer risk is still uncertain, ${ }^{19-21}$ the fourth sensitivity analysis was conducted to evaluate cancer risk of therapies involving lithium and valproate prescribed alone, in combination, or one of these two drugs followed sequentially by the other. Fifth, a number of studies have investigated the relationship between cancer and psychiatric agents, such as TCAs, SSRIs and SGAs, ${ }^{15,22-25}$ therefore, these psychiatric agents may have confounded our results. We performed backward selection to find the most and least significant psychiatric drug variables in the full model, and we analysed the cancer risk in each permutation.

To assess whether the overall risk of cancer varied across patient characteristics, we performed analyses stratified by subgroups of various characteristics of the patients, including age and the Charlson Comorbidity Index (CCI).

\section{Results}

We identified 4729 patients with bipolar disorder, of which $3250(68.7 \%)$ were anticonvulsant-only users, $370(7.8 \%)$ were lithium-only users and $1109(23.5 \%)$ were lithium and anticonvulsants users. Median follow-up time for the lithium-only group was 7.1 (interquartile range $(\mathrm{IQR})=4.7-9.9)$ years, for the lithium and anticonvulsants group it was $7.5(\mathrm{IQR}=5.1-9.9)$ years and for the anticonvulsant-only group it was 5.2 $(\mathrm{IQR}=3.1-7.8)$ years. The majority $(97.6 \%)$ of patients were censored by the end of the study period, followed by death and disenrolment (Table 1). In total 115 cases of cancer were identified, and the mean (s.d.) age of cancer incidence in our study cohort was 60.7 (15.3) years. There were 86 individuals (2.65\%) with newly diagnosed cancer during follow-up (4.74 cases per 1000 person-years) in the anticonvulsant-only group and 29 individuals (1.96\%) with newly diagnosed cancer during follow-up (2.66 cases per 1000 person-years) in the combined lithium with or without anticonvulsants group (online Table DS3).

Baseline characteristics are compared in Table 1 (see online Table DS4 for a more detailed version of this table). Patients taking lithium were younger than the anticonvulsant-only group $(P<0.001)$. The CCI indicated lower scores for those taking lithium $(P<0.001)$. Although the prevalence rates of physical comorbidities were lower in those taking lithium, the prevalence rates of psychiatric comorbidities were higher in individuals with lithium exposure (except for anxiety) (online Table DS4).

Separate Cox regressions were implemented to investigate the hazard ratios of cancer risks in the groups (Table 2). The unadjusted hazard ratios were 0.426 (95\% CI $0.186-0.975$ ) in the lithium-only group and 0.511 (95\% CI $0.322-0.812$ ) in the lithium and anticonvulsant group, using the anticonvulsantonly group as the reference. Because the sample size of the lithium-only group was small, we also computed the hazard ratio for the combined group using lithium with or without anticonvulsants; the hazard ratio was 0.491 (95\% CI 0.321-0.750). After being weighted using the IPTW approach (Model I) and being additionally adjusted for other chemoprevention drugs (Model II), only the hazard ratio of the lithium and anticonvulsant group (Model I: 0.628, 95\% CI 0.468-0.843; Model II: 0.669, 95\% CI $0.488-0.915)$ and the combined lithium with or without anticonvulsants group (Model I: 0.723, 95\% CI 0.557-0.940; Model II: $0.735,95 \%$ CI $0.554-0.974)$ remained statistically significant. Although the number of cancer cases was not large in Table 2, we conducted post hoc power analyses of significant hazard ratios and found that most powers were higher than $80 \%$; four hazard ratios $(0.723,0.735,0.556$ and 0.552$)$ were between 71 and $78 \%$. For site-specific cancer, lithium users showed trends of decreased cancer risks, except for bone, skin, and connective and other soft tissue cancer risks ( $\mathrm{HR}=3.012,95 \%$ CI $0.798-11.365)$ and

\begin{tabular}{|c|c|c|c|c|}
\hline & $\begin{array}{l}\text { Anticonvulsant-only group } \\
\qquad(n=3250)\end{array}$ & $\begin{array}{l}\text { Lithium-only group } \\
\qquad(n=370)\end{array}$ & $\begin{array}{l}\text { Lithium-anticonvulsant group } \\
\qquad(n=1109)\end{array}$ & $P$ \\
\hline Follow-up, years: median, IQR & $5.2(3.1-7.8)$ & $7.1(4.7-9.9)$ & $7.5(5.1-9.9)$ & $<0.001$ \\
\hline Cancer event, $n$ (\%) & $86(2.6)$ & $6(1.6)$ & $23(2.1)$ & 0.324 \\
\hline \multicolumn{5}{|l|}{ Censored } \\
\hline Death & $163(5.0)$ & $14(3.8)$ & $56(5.0)$ & \\
\hline Disenrolment & $87(2.7)$ & $11(3.0)$ & $13(1.2)$ & \\
\hline End of study period & $2914(89.7)$ & 339 (91.6) & 1017 (91.7) & \\
\hline Male, $n(\%)$ & $1327(40.8)$ & $159(43.0)$ & $480(43.2)$ & 0.328 \\
\hline Age, mean (s.d.) & $45.9(17.5)$ & $38.5(13.9)$ & $37.1(13.7)$ & $<0.001$ \\
\hline Median, IQR & $44(31-59)$ & 37.5 (27-47) & $35(26-46)$ & $<0.001$ \\
\hline \multicolumn{5}{|c|}{ Charlson Comorbidity Index, n (\%) } \\
\hline 0 & $2116(65.1)$ & $303(81.9)$ & $870(78.4)$ & $<0.001$ \\
\hline 1 & $638(19.6)$ & $46(12.4)$ & $161(14.5)$ & \\
\hline 2 & $267(8.2)$ & $15(4.1)$ & $41(3.7)$ & \\
\hline$\geqslant 3$ & $229(7.0)$ & $6(1.6)$ & $37(3.3)$ & \\
\hline Mean (s.d.) & $0.63(1.11)$ & $0.26(0.66)$ & $0.34(0.80)$ & $<0.001$ \\
\hline
\end{tabular}




\begin{tabular}{|c|c|c|c|c|c|}
\hline & Patients, $n$ & $\begin{array}{l}\text { Cancer } \\
\text { case, } n\end{array}$ & $\begin{array}{l}\text { Unadjusted, HR } \\
\qquad(95 \% \mathrm{Cl})\end{array}$ & $\begin{array}{l}\text { Model I, adjusted } \\
\text { HR }(95 \% \mathrm{Cl})\end{array}$ & $\begin{array}{c}\text { Model II, adjusted } \\
\text { HR }(95 \% \mathrm{Cl})\end{array}$ \\
\hline Anticonvulsants only (referent) & 3250 & 86 & 1.000 & 1.000 & 1.000 \\
\hline Lithium only & 370 & 6 & $0.426(0.186-0.975)$ & $0.994(0.695-1.421)$ & $0.891(0.613-1.295)$ \\
\hline Lithium and anticonvulsants & 1109 & 23 & $0.511(0.322-0.812)$ & $0.628(0.468-0.843)$ & $0.669(0.488-0.915)$ \\
\hline Anticonvulsants only (referent) & 3250 & 86 & 1.000 & 1.000 & 1.000 \\
\hline Lithium with or without anticonvulsants & 1479 & 29 & $0.491(0.321-0.750)$ & $0.723(0.557-0.940)$ & $0.735(0.554-0.974)$ \\
\hline \multicolumn{6}{|l|}{ Cumulative dose of lithium (CDDD) ${ }^{\mathrm{b}}$} \\
\hline$<28$ & 460 & 7 & $0.431(0.199-0.931)$ & $0.801(0.551-1.164)$ & $0.762(0.516-1.125)$ \\
\hline $28-215$ & 518 & 11 & $0.517(0.276-0.971)$ & $0.828(0.588-1.166)$ & $0.919(0.640-1.318)$ \\
\hline$>215$ & 501 & 11 & $0.510(0.272-0.958)$ & $0.556(0.374-0.825)$ & $0.552(0.367-0.831)$ \\
\hline$P$-value for trend & & & 0.0044 & 0.0050 & 0.0130 \\
\hline \multicolumn{6}{|l|}{ Average daily $D D D^{b, c}$} \\
\hline$<0.68$ & 661 & 15 & $0.646(0.373-1.190)$ & $0.941(0.688-1.287)$ & $0.935(0.671-1.302)$ \\
\hline $0.68-0.90$ & 424 & 8 & $0.442(0.214-0.914)$ & $0.753(0.520-1.090)$ & $0.727(0.494-1.070)$ \\
\hline$>0.90$ & 394 & 6 & $0.336(0.147-0.770)$ & $0.377(0.219-0.604)$ & $0.425(0.252-0.716)$ \\
\hline$P$-value for trend & & & 0.0007 & 0.0002 & 0.0008 \\
\hline \multicolumn{6}{|c|}{$\begin{array}{l}\text { HR, hazard ratio; DDD, defined daily dose. } \\
\text { a. Model I: weighted by inverse probability of treatment weights (IPTW; Model II: weighted by IPTW and adjusted for the usage of non-steroidal anti-inflammatory drugs, aspirin, } \\
\text { cyclo-oxygenase-2 inhibitors, angiotensin-converting enzyme inhibitors/angiotensin receptor blockers, metformin, sulfonylurea, systemic steroid and statin. } \\
\text { b. Referent group: never-users of lithium. } \\
\text { c. Average daily DDD is the cumulative defined daily dose divided by the cumulative prescription days. }\end{array}$} \\
\hline
\end{tabular}

genitourinary cancer risk $(\mathrm{HR}=1.014,95 \%$ CI $0.472-2.179$, online Table DS3).

When considering the dose-response of lithium usage, there were significant trends of reduced overall cancer risk with increasing cumulative dose of lithium ( $P$-value for trends $<0.05$; Table 2). Users of lithium were divided into three tertiles by cumulative dose of lithium (cDDD) and average daily DDD. Significantly lower risks were found in the third tertile of cDDD (cDDD $>215, \mathrm{HR}=0.552,95 \%$ CI $0.367-0.831)$ and average daily DDD $(\mathrm{DDD}>0.9, \mathrm{HR}=0.425,95 \%$ CI $0.252-0.716$ ).

The sensitivity analyses consistently showed a reduced risk of overall cancer for lithium users $v$. anticonvulsant-only users (Table 3 ). In the broader definition of cancer diagnosis, the hazard ratio was close to the primary analysis $(\mathrm{HR}=0.764,95 \%$ CI $0.624-$ 0.936). After restricting the analysis to those with psychiatrists' prescriptions in both drug groups, the HR estimates were slightly lower than those of the primary analysis $(\mathrm{HR}=0.684,95 \% \mathrm{CI}$ 0.503-0.929) (Table 3). In the analysis excluding patients with epilepsy prior to the index date, lithium users showed a trend of decreased cancer risk, but it was non-significant $(\mathrm{HR}=0.767$, 95\% CI 0.572-1.028). In the fourth analysis, valproate users or lithium users had similarly lower cancer risks $(\mathrm{HR}=0.848,95 \%$ CI $0.563-1.277$ for valproate users; $\mathrm{HR}=0.777,95 \%$ CI $0.511-$ 1.181 for lithium users). There was a significantly decreased overall cancer risk in the lithium and valproate combined or sequential therapy group $(\mathrm{HR}=0.584,95 \% \mathrm{CI} 0.386-0.885)$. In the fifth analysis, we chose to analyse the cancer risk related to lithium, SGA and hypnotics because patients had exposure to these agents. The hazard ratio of cancer risk for both SGA/hypnotics and lithium/SGA/hypnotics combinations had statistically lower hazard ratios $(\mathrm{HR}=0.444,95 \%$ CI $0.254-0.778$ for SGA/hypnotics; $\mathrm{HR}=0.305,95 \%$ CI $0.173-0.538$ for lithium/SGA/hypnotics). The hazard ratio for the lithium/SGA/hypnotics treatment was lower than that for SGA/hypnotics treatment, supporting our finding that lithium has a protective effect on overall cancer risk.

In the subgroup analysis of age groups, lithium users between 18 and 44 years of age (HR $=0.570,95 \%$ CI $0.339-0.959)$ were associated with decreased cancer risk (Table 4). Similarly, patients without comorbidities $(\mathrm{CCI}=0)$ had a significant protective effect with lithium usage.

\section{Discussion}

\section{Main findings}

Previous studies have shown that patients with bipolar disorder are at increased risk for cancer. ${ }^{26,27}$ In the Taiwan cancer registry, the crude cancer incidence rates during our study duration (19992010) were 2.55 to 4.00 per 1000 person-years. ${ }^{28}$ The cancer incidence rates in our study cohort of 4.74 cases per 1000 person-years in the anticonvulsant-only group and 2.66 cases per 1000 person-years in the lithium with or without anticonvulsants group (online Table DS3) are consistent with previous reports that patients with bipolar disorder are at increased risk for cancer. We demonstrate that lithium usage in patients with bipolar disorder is associated with a $26.5 \%$ lower risk $(\mathrm{HR}=0.735,95 \%$ CI 0.554-0.974) of overall cancer, and a cDDD of more than 215 is associated with a $44.8 \%$ lower risk $(\mathrm{HR}=0.552,95 \% \mathrm{CI}$ $0.367-0.831$ ). To our knowledge, this is the first population-based study to demonstrate overall reduced cancer risk in patients with bipolar disorder taking lithium.

Lithium was described in 1996 as an inhibitor of GSK-3. ${ }^{4}$ Previous research regarding the association of GSK-3 with cancer was limited in in vivo and in vitro studies. Although it may not be feasible to conduct clinical studies to investigate the association of lithium usage and cancer development, the availability of largescale population-based data has become a useful resource to investigate possible chemoprevention effects in the population. One previous hospital-based study involving psychiatric patients showed a lower but non-significant risk $(\mathrm{OR}=0.79,95 \% \mathrm{CI}$ 0.17-3.60) of cancer of non-epithelial origin in the lithium group. ${ }^{6}$ Although we were able to report hazard ratios from different types of cancer, one still needs to interpret results with caution because the number of specific cancer cases was low and there may not be enough statistical power. The duration of bipolar disorder treatment is generally from 6 months to 3 years, depending on individual clinical considerations. ${ }^{29,30}$ Long-term exposure is an important drug safety issue. Our study showed that the protective effect of lithium only exists when the CDDD in the highest tertile is reached. Nevertheless, we did not see a similar finding for cumulative prescription days. We suspected that there are two exposure situations, higher daily dose with shorter duration and 


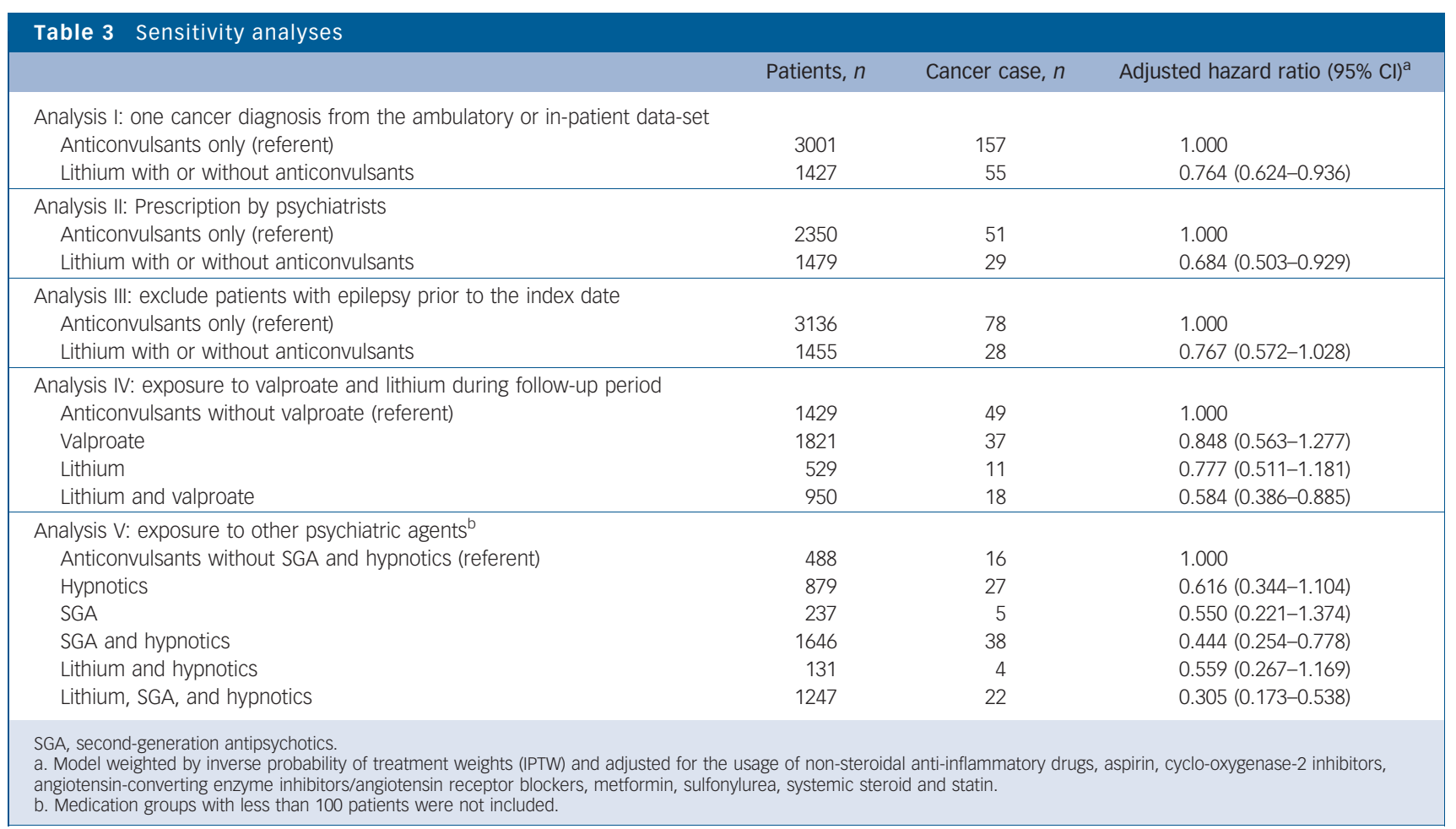

\begin{tabular}{|c|c|c|c|}
\hline & Patients, $n$ & Cancer case, $n$ & Adjusted hazard ratio $(95 \% \mathrm{Cl})^{c}$ \\
\hline \multicolumn{4}{|l|}{ Subgroup analysis by age groups } \\
\hline \multicolumn{4}{|l|}{$18-44$ years old } \\
\hline Anticonvulsants only (referent) & 1638 & 17 & 1.000 \\
\hline Lithium with or without anticonvulsants & 1070 & 12 & $0.570(0.339-0.959)$ \\
\hline \multicolumn{4}{|l|}{$45-59$ years old } \\
\hline Anticonvulsants only (referent) & 838 & 28 & 1.000 \\
\hline Lithium with or without anticonvulsants & 291 & 13 & $0.824(0.528-1.285)$ \\
\hline \multicolumn{4}{|l|}{$\geqslant 60$ years old } \\
\hline Anticonvulsants only (referent) & 774 & 41 & 1.000 \\
\hline Lithium with or without anticonvulsants & 118 & 4 & $1.316(0.702-2.466)$ \\
\hline \multirow{2}{*}{\multicolumn{4}{|c|}{$\begin{array}{l}\text { Subgroup analysis by Charlson Comorbidity Index (CCl) } \\
\mathrm{CCl}, 0\end{array}$}} \\
\hline & & & \\
\hline Anticonvulsants only (referent) & 2116 & 43 & 1.000 \\
\hline Lithium with or without anticonvulsants & 1173 & 18 & $0.581(0.396-0.853)$ \\
\hline \multicolumn{4}{|l|}{$\mathrm{CCl}, 1$} \\
\hline Anticonvulsants only (referent) & 638 & 21 & 1.000 \\
\hline Lithium with or without anticonvulsants & 207 & 7 & $0.713(0.378-1.346)$ \\
\hline \multicolumn{4}{|l|}{$\mathrm{CCl} \geqslant 2$} \\
\hline Anticonvulsants only (referent) & 496 & 22 & 1.000 \\
\hline Lithium with or without anticonvulsants & 99 & 4 & $1.104(0.576-2.118)$ \\
\hline
\end{tabular}

lower daily dose with longer duration. An average daily dose assessment was further performed and the dose-response relationship was proven. However, more research is still needed to investigate the cumulative dose and days to reach the effect.

As lithium users were younger and had lower CCIs, age and CCI may be important confounders in our results. Only in the youngest stratification and lowest CCI stratification were the positive effects of lithium for cancer risk shown. This may result from there not being enough power in the subgroup analysis. Nevertheless, the hazard ratio of every subgroup showed a trend of lower overall cancer risk, except for patients older than 60 years.

As there are several medication options for bipolar disorder treatment, it is important to evaluate whether they differ with regard to the risk of cancer. Valproate is one of the anticonvulsants used for bipolar disorder. The effects of valproate on GSK-3 have been extensively studied, but there are no consistent conclusions. This discrepancy possibly results from minor differences in substrates or assay conditions. ${ }^{31}$ Valproate has been reported to act as a histone deacetylase (HDAC) inhibitor, which may be associated with several haematological malignancies. Several preclinical studies support the anticarcinogenic effects of valproate, although conclusions in epidemiological studies are inconsistent. ${ }^{19-21}$ We restricted the analysis of anticonvulsants to valproate and evaluated the effect of valproate in our sensitivity study. Lithium-valproate exposure has an additional effect for lower overall risk of cancer compared with either lithium or valproate exposure in patients with bipolar disorder. Our finding supports the idea that lithium has some protective effect regardless 
of valproate. There were also many other psychiatric agents that were prescribed during the follow-up period that may interfere with the results. We found that SGA and hypnotics use in the follow-up period reduced overall cancer risk. Moreover, lithium/ SGA/hypnotic users had a lower risk than SGA/hypnotics users. These findings enhanced the idea of a protective effect from lithium despite the fact that a protective effect may also be derived from other psychiatric agents.

\section{Limitations}

The present study has some limitations that warrant attention. First, possible misclassification may occur when extracting patients only from records of an ambulatory and in-patient care database. In this study, the diagnoses of cancer were confirmed by linking to the catastrophic illness database. The registration of cancer as a catastrophic illness is very rigorous and has to be approved by evaluating pathology and/or cytology evidence. Hence, the catastrophic illness database has been widely used in previous studies to ascertain cancer events. ${ }^{26,27}$ Although bipolar disorder may be misdiagnosed, the probability was less likely because all prescriptions should have a corresponding diagnosis in the rigorous claims review system, which is the main indicator of lithium for bipolar disorder. Anticonvulsants have a very diverse range of indications. Two sensitivity analyses (limited to prescriptions by psychiatrists and excluding patients with epilepsy) were performed to strengthen our results regarding patients with bipolar disorder. In the analysis excluding patients with epilepsy prior to the index date, lithium users showed a non-significant trend towards decreased cancer risk $(\mathrm{HR}=0.767$, 95\% CI 0.572-1.028). This supports the finding that the indications for anticonvulsants in our study cohort may affect the hazard ratio. However, this would not affect the conclusion of a positive effect of lithium on overall cancer risk.

Second, cancer may develop over a long period of time and most observational studies, including this study, do not include a sufficient follow-up period. Abnormal symptoms may be present several years before a definite diagnosis. However, the same definition is applied to both comparison groups; therefore, the possible misclassification may only have a limited effect on the risk. We adopted a non-strict definition of diagnosis, and the results demonstrated that lithium users had a lower risk of early abnormal signs. The results could be evidence of a lithium effect. However, there is still a need for more studies with longer follow-up to strengthen our conclusion.

Third, because the purpose of the NHI database is reimbursement, there is no information on risk factors for cancer, such as family history, body mass index, smoking or any laboratory parameters. We used patient comorbidities as surrogates to adjust for patient health status and lifestyle in our study. Although there are other unmeasured confounders, we believe the methodology used in this study is robust. Fourth, the actual amount of drugs taken could not be determined from the database. Therefore, our conclusion is based on reasonable adherence with medication use. Fifth, there is no histopathological information in the LHID data-set, therefore we were not able to investigate this issue. A previous study indicated that the association between lithium and cancer risk may depend on the type of cancer., ${ }^{2,6}$

Finally, we may not have identified all individuals taking psychiatric medications. However, psychiatric agents are not over-the-counter drugs. Moreover, bipolar disorder is one of the chronic psychoses on the Registry for Catastrophic Illness in Taiwan. When patients were approved by the Registry, the disease-specific treatment cost was covered by the NHI programme, and no copayment for the ambulatory visit or for in-patient care was required. For these reasons, we anticipate that the probability of patient-used self-paid psychiatric agents is very low. Furthermore, we believe most drug-exposure information in our study cohort is completely documented in the database, even though the data-set does not contain information on out-of-pocket treatments.

\section{Implications}

The present findings imply that lithium use was associated with a lower incidence of overall cancer risk in patients with bipolar disorder. There is a dose-response relationship for lithium use and a higher cumulative dose was associated with significant risk reduction in overall cancer risk. Further studies are needed that focus on the impact on different types of cancer.

\section{Ru-Yu Huang, MSc, School of Pharmacy, Master Program in Clinical Pharmacy, Kaohsiung Medical University, Kaohsiung; Kun-Pin Hsieh, PharmD, PhD, School of Pharmacy, College of Pharmacy, Kaohsiung Medical University and Department of Pharmacy, Kaohsiung Medical University Hospital, Kaohsiung; Wan-Wen Huang, MSc, School of Pharmacy, Master Program in Clinical Pharmacy, Kaohsiung Medical University, Kaohsiung; Yi-Hsin Yang, PhD, School of Pharmacy, College of Pharmacy, Kaohsiung Medical University, Kaohsiung, Taiwan}

Correspondence: Yi-Hsin Yang, PhD, School of Pharmacy, College of Pharmacy, Kaohsiung Medical University, 100, Shih-Chuan 1st Road, Kaohsiung, 807, Taiwan. Email: yihsya@kmu.edu.tw

First received 2 Aug 2015, final revision 11 Feb 2016, accepted 12 Feb 2016

\section{References}

1 Takahashi-Yanaga F. Activator or inhibitor? GSK-3 as a new drug target. Biochem Pharmacol 2013; 86: 191-9.

2 McCubrey JA, Davis NM, Abrams SL, Montalto G, Cervello M, Basecke J, et al. Diverse roles of GSK-3: tumor promoter-tumor suppressor, target in cancer therapy. Adv Biol Regul 2014; 54: 176-96.

3 McCubrey JA, Steelman LS, Bertrand FE, Davis NM, Sokolosky M, Abrams SL, et al. GSK-3 as potential target for therapeutic intervention in cancer. Oncotarget 2014; 5: 2881-911.

4 Brown KM, Tracy DK. Lithium: the pharmacodynamic actions of the amazing ion. Ther Adv Psychopharmacol 2013; 3: 163-76.

5 Severus E, Taylor MJ, Sauer C, Pfennig A, Ritter P, Bauer M, et al. Lithium for prevention of mood episodes in bipolar disorders: systematic review and meta-analysis. Int J Bipolar Disord 2014; 2: 1-17.

6 Cohen Y, Chetrit A, Cohen Y, Sirota P, Modan B. Cancer morbidity in psychiatric patients: influence of lithium carbonate treatment. Med Oncol 1998; 15: 32-6.

7 Cheng $\mathrm{CL}$, Kao YH, Lin SJ, Lee CH, Lai ML. Validation of the National Health Insurance Research Database with ischemic stroke cases in Taiwan. Pharmacoepidemiol Drug Saf 2011; 20: 236-42.

8 National Health Insurance Research Database, Taiwan. Data Subset. NHIRD, 2015 (http://nhird.nhri.org.tw/en/Data_Subsets.html).

9 World Health Organization. International Statistical Classification of Diseases and Related Health Problems (ICD-9). WHO, 1978.

10 Young LT. What exactly is a mood stabilizer? J Psychiatry Neurosci 2004; 29: 87-8.

11 Bowden CL. Anticonvulsants in bipolar disorders: current research and practice and future directions. Bipolar Disord 2009; 11: 20-33.

12 WHO Collaborating Centre for Drug Statistics Methodology. ATC/DDD Index 2015. WHOCC, 2015 (http://www.whocc.no/atc_ddd_index/).

13 Deyo RA, Cherkin DC, Ciol MA. Adapting a clinical comorbidity index for use with ICD-9-CM administrative databases. J Clin Epidemiol 1992; 45: 613-9.

14 Gerhard T, Devanand DP, Huang C, Crystal S, Olfson M. Lithium treatment and risk for dementia in adults with bipolar disorder: population-based cohort study. Br J Psychiatry 2015; 207: 46-51.

15 Pottegard A, Friis S, Andersen M, Hallas J. Use of benzodiazepines or benzodiazepine related drugs and the risk of cancer: a population-based case-control study. Br J Clin Pharmacol 2013; 75: 1356-64.

16 Brookhart MA, Wyss R, Layton JB, Sturmer T. Propensity score methods for confounding control in nonexperimental research. Circ Cardiovasc Qual Outcomes 2013; 6: 604-11.

17 Austin PC. The performance of different propensity score methods for estimating marginal hazard ratios. Stat Med 2013; 32: 2837-49. 
18 Kaae J, Carstensen L, Wohlfahrt J, Melbye M, Boyd HA. Epilepsy, antiepileptic medication use and risk of cancer. Int J Cancer 2014; 134: 932-8.

19 Singh G, Bell GS, Driever PH, Sander JW. Cancer risk in people with epilepsy using valproate-sodium. Acta Neurol Scand 2012; 125: 234-40.

20 Hallas J, Friis S, Bjerrum L, Stovring H, Narverud SF, Heyerdahl T, et al. Cancer risk in long-term users of valproate: a population-based case-control study. Cancer Epidemiol Biomarkers Prev 2009; 18: 1714-9.

21 Kang $\mathrm{H}$, Gillespie TW, Goodman M, Brodie SA, Brandes M, Ribeiro M, et al. Long-term use of valproic acid in US veterans is associated with a reduced risk of smoking-related cases of head and neck cancer. Cancer 2014; 120 : 1394-400.

22 Boursi B, Lurie I, Mamtani R, Haynes K, Yang YX. Anti-depressant therapy and cancer risk: a nested case-control study. Eur Neuropsychopharmacol 2015; 25: 1147-57.

23 Lee HK, Eom CS, Kwon YM, Ahn JS, Kim S, Park SM. Meta-analysis: selective serotonin reuptake inhibitors and colon cancer. Eur J Gastroenterol Hepatol 2012; 24: 1153-7.

24 Walker AJ, Card T, Bates TE, Muir K. Tricyclic antidepressants and the incidence of certain cancers: a study using the GPRD. Br J Cancer 2011; 104 193-7.

25 Fond G, Macgregor A, Attal J, Larue A, Brittner M, Ducasse D, et al. Antipsychotic drugs: pro-cancer or anti-cancer? A systematic review. Med Hypotheses 2012; 79: 38-42.
26 Lin GM, Chen YJ, Kuo DJ, Jaiteh LE, Wu YC, Lo TS, et al. Cancer incidence in patients with schizophrenia or bipolar disorder: a nationwide population-based study in Taiwan, 1997-2009. Schizophr Bull 2013; 39 $407-16$

27 Hung $\mathrm{Y}-\mathrm{N}$, Yang S-Y, Huang M-C, Lung F-W, Lin S-K, Chen K-Y, et al. Cancer incidence in people with affective disorder: nationwide cohort study in Taiwan, 1997-2010. Br J Psychiatry 2014; 205: 183-8.

28 Health Promotion Administration, Ministry of Health and Welfare. Cancer Registry Annual Report 2010, Taiwan. Health Promotion Administration, Ministry of Health and Welfare, 2010 (http://www.hpa.gov.tw/BHPNet/Web/ Stat/StatisticsShow.aspx?No $=200911300001$ ).

29 Grunze H, Vieta E, Goodwin GM, Bowden C, Licht RW, Moller HJ, et al. The World Federation of Societies of Biological Psychiatry (WFSBP) guidelines for the biological treatment of bipolar disorders: update 2012 on the long-term treatment of bipolar disorder. World J Biol Psychiatry. 2013; 14: 154-219.

30 Bai YM, Chang CJ, Tsai SY, Chen YC, Hsiao MC, Li CT, et al. Taiwan consensus of pharmacological treatment for bipolar disorder. J Chin Med Assoc 2013; 76: 547-56.

31 Rowe MK, Wiest C, Chuang DM. GSK-3 is a viable potential target for therapeutic intervention in bipolar disorder. Neurosci Biobehav Rev 2007; 31 920-31.

\section{reflection}

\section{Reflection on Richard Lazarus' Emotion and Adaptation}

\section{Ayat Ali}

Emotion and Adaptation, first published in 1991, is a thorough and scholarly book hypothesising a cognitive theory of emotion Lazarus has been a pioneer in this field for the past 40 years; he argues that emotions have intentionality and that their significance and force are determined by our cognition. This then creates a judgement as to whether or not we can cope with the external event or situation, which forms an emotional reaction.

Lazarus' book helps us understand why emotions are so meaningful to the human race. Drawing together all the related research, it is a centrepiece of work for all those interested in emotion and cognition and applicable to sociologists and psychologists as well as psychiatrists. Lazarus' ideas have attracted my attention as they are, relatively speaking, radical. In today's world the reductionist perspective has dominated explanations for the wonders of the human mind. Mental health conditions have been reduced to chemical imbalances and a pathological structure of the brain, stripping the mind and emotions of all higher meanings.

Lazarus proposes a multidimensional appraisal theory of emotion, where an appraisal is an evaluation of an external event. His theory of emotion can be broken down into a sequence: (1) cognitive appraisal, (2) physiological response, and (3) action. Lazarus endeavoured to answer two questions, the nature of the appraisals which underlie each emotional reaction and the determining antecedent conditions of these appraisals. The answers to these questions would help us understand what emotion is, why we have certain emotions in particular situations and why emotional reactions vary from person to person even in the same or similar situations.

Lazarus' theory merges well with other schools of thought: the somatic theory, the cognitive theory and Skinner's behavioural theory. One of the strengths of the appraisal theory is that it explains the variation of reactions among people subject to the same event. Primary appraisal is the chief factor influencing whether or not an individual can cope in a stressful situation. In contrast, Robert Zajonc states that cognitive processes and affect are independent of each other. Affect precedes cognition and is far more influential; he calls this 'the primacy of affect'. He argued that the affect response could be made sooner than the cognitive response. Zajonc uses the example that infants and animals can make facial expressions highly indicative of emotion with no preceding cognition; this however, cannot be proved.

I cannot help but agree with both Lazarus and Zajonc. I do not believe that their theories are mutually exclusive. I would argue that we could place emotions on a continuum from least cognitive to highly cognitive, basic emotions to complex emotions. Zajonc's theories are most likely to hold true and be the dominant system for lower organisms. Paul Ekman gives a nice analogy of emotions being like primary colours, primary emotions blending into more complex emotions to give the full spectrum of human emotional experience. 